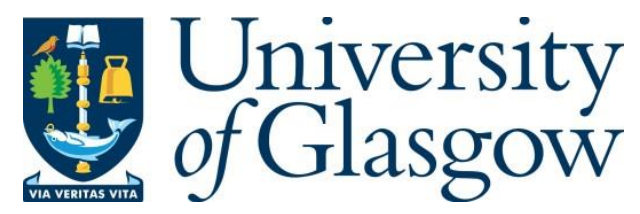

Wilson, G., McGill, M., Jamieson, M., Williamson, J. R. and Brewster, S. A. (2018) Object Manipulation in Virtual Reality Under Increasing Levels of Translational Gain. In: 2018 CHI Conference on Human Factors in Computing Systems, Montréal, QC, Canada, 21-26 Apr 2018, p. 99. ISBN 9781450356206.

There may be differences between this version and the published version. You are advised to consult the publisher's version if you wish to cite from it.

(C) The Authors 2018. This is the author's version of the work. It is posted here for your personal use. Not for redistribution. The definitive Version of Record was published in the 2018 CHI Conference on Human Factors in Computing Systems, Montréal, QC, Canada, 21-26 Apr 2018, p. 99. ISBN 9781450356206, https://doi.org/10.1145/3173574.3173673.

http://eprints.gla.ac.uk/156185/

Deposited on: 23 January 2018

Enlighten - Research publications by members of the University of Glasgow http://eprints.gla.ac.uk 


\title{
Object Manipulation in Virtual Reality Under Increasing Levels of Translational Gain
}

\author{
Graham Wilson*, Mark McGill*, Matthew Jamieson, Julie R. Williamson, Stephen A. Brewster \\ Glasgow Interactive Systems Section \\ University of Glasgow \\ Glasgow, Scotland \\ \{first.last\}@glasgow.ac.uk http://mig.dcs.gla.ac.uk
}

\begin{abstract}
Room-scale Virtual Reality (VR) has become an affordable consumer reality, with applications ranging from entertainment to productivity. However, the limited physical space available for room-scale VR in the typical home or office environment poses a significant problem. To solve this, physical spaces can be extended by amplifying the mapping of physical to virtual movement (translational gain). Although amplified movement has been used since the earliest days of VR, little is known about how it influences reach-based interactions with virtual objects, now a standard feature of consumer VR. Consequently, this paper explores the picking and placing of virtual objects in VR for the first time, with translational gains of between $1 \mathrm{x}$ (a one-to-one mapping of a $3.5 \mathrm{~m} * 3.5 \mathrm{~m}$ virtual space to the same sized physical space) and $3 \mathrm{x}(10.5 \mathrm{~m} * 10.5 \mathrm{~m}$ virtual mapped to $3.5 \mathrm{~m} * 3.5 \mathrm{~m}$ physical). Results show that reaching accuracy is maintained for up to $2 \mathrm{x}$ gain, however going beyond this diminishes accuracy and increases simulator sickness and perceived workload. We suggest gain levels of $1.5 \mathrm{x}$ to $1.75 \mathrm{x}$ can be utilized without compromising the usability of a VR task, significantly expanding the bounds of interactive room-scale VR.
\end{abstract}

\section{Author Keywords}

Virtual Reality; amplified movement; object manipulation; translational gain; redirected walking.

\section{ACM Classification Keywords}

H.5.m. Information interfaces and presentation (e.g., HCI): Miscellaneous;

\section{INTRODUCTION}

Virtual environments (VEs) provide limitless opportunities constrained only by the creator's imagination. In practice, VEs that are intended to be explored by real-world walking are severely limited by the physical space available in the offices and homes [41] where commercial Virtual Reality

Library copy
(VR) headsets are being used. A 1:1 mapping of physical to virtual movement requires VEs to fit the constraints of the physical space available. This significantly limits the possibilities for VE design and novel VR applications, reminds users of the physical constraints of their setup and breaks immersion when physical boundaries interrupt use.

A number of approaches have been used to overcome physical space constraints. Amplified movements allow users to traverse larger virtual spaces by accelerating or amplifying the mapping of physical to virtual movement [22,43,44,57]. This is commonly referred to as translational gain, a multiplier on the $x / z$ position of the VR user, where walking $1 \mathrm{~m}$ in physical space can lead to walking $2 \mathrm{~m}(2.0 \mathrm{x}$ gain) to $50 \mathrm{~m}$ (50.0x gain) in virtual space. This technique requires no training and can enable larger singular virtual rooms whose extents are fully reachable and spatially consistent, with every point in VR mapped to a point in real-world space.

Translational gain has frequently been overlooked in favour of both redirected walking and locomotion interfaces. Redirected walking uses combinations of translational [21], rotational [27] and curvature [28] gain alongside other perceptual tricks to portray infinitely traversable virtual spaces. However, these techniques have significant limitations regarding physical requirements (minimum $6 \mathrm{~m}^{2}$ [45] but realistically up room-scale to $22 \mathrm{~m}^{2}$ [19]), and they cannot support singleroom virtual spaces where every visible corner is consistently reachable and fully explorable. Virtual locomotion techniques [54] such as teleportation, walking in-place and flight also allow for infinite exploration, but they require training, potentially increase simulator sickness [25] and undermine the spatial understanding of the virtual space [58]. Omni-directional treadmills [12] allow for effortful physical traversal whilst remaining stationary in reality, but require costly additional hardware, and may restrain movement and interaction.

Consequently, this paper examines how translational gain can be applied to interactive room-scale VR for the first time. Translational gain has the greatest potential to impact everyday VR users across a range of physical environments. It costs nothing, is trivial to apply, and allows for singular, complex, fully explorable spaces (e.g. small shop, kitchen) to be approximated within the limited physical bounds of a 
home or office. It requires no additional user training or interaction. However, VR experiences are now typically highly interactive. Grasping and manipulating virtual objects is supported by default in many consumer VR experiences, typically enacted via handheld controllers (such as those bundled with the HTC Vive [20]) or optical hand tracking (e.g. Leap Motion [30]). Given this, one key unknown issue, not addressed in earlier work, is how amplified movement impacts interaction and manipulation of virtual objects: a fundamental part of interaction in VR. For example, does amplified movement cause users to overshoot when reaching for objects, misjudge distances relative to their bodies, or collide with virtual barriers? As off-the-shelf input techniques with handheld controllers and positional tracking of VR head-sets become the standard setup, new models of amplified movement must be developed that take this whole-body interaction into account.

In this paper, we examine two key questions about virtual object interaction under varying levels of translational gain. Firstly, to what extent does translational gain impair a user's ability to interact with, and manipulate, virtual objects. Secondly, what are the resultant maximal usable levels of translational gain that can be applied, given a virtual environment that requires frequent object manipulations. We do so under the constraint of providing a fully reachable, freely explorable space where each point in the virtual space maps to a specific point in the real world, meaning no dynamic/variable translational or rotational gain. In a study $(n=20)$, we measured how precisely participants could reach for, pick up and place virtual objects at varying positions and heights, and walk routes around virtual obstacles, under varying levels of translational gain. From this, we present new guidelines for applying translational gain to both highly interactive VE's, and environments where object manipulation does not require stretching/reaching/bending.

\section{RELATED RESEARCH}

A range of methods has been developed to enable VR users to traverse virtual environments larger than the physical space available to them. Movement can be simulated from a fixed position using virtual movement or by physically moving within a tracked space using redirected walking and movement gain

\section{Virtual Movement}

The current position and point of view (POV) of the user can be instantaneously transported to another position in the VE using controls such as buttons or pointers [54], but the sudden change in position can cause disorientation [5] and can diminish sense of presence [6]. Virtual movement can also be continuous like a flyover [50], where a joystick controls continuous movement through a VE. These approaches are fully supported in VR software development kits and are used in many popular games. However, in all cases of virtual movement, seeing movement without feeling physical movement is a major cause of simulator sickness [25].
Previous work has explored ways of adding physicality to virtual movement without actually walking. Tregillus et al. [48] explored how head tilt could be used to navigate in a VE. However, such approaches have notable problems regarding drift and the potential for increased simulator sickness. Wilson et al. [55] compared walking-in-place and armswinging-in-place with actually walking. Although walkingin-place performed better than arm-swinging-in-place in terms of spatial awareness, neither was a good as physically walking [55]. Whilst walking-in-place can add more realism and a sensation of physical activity, it still suffers from a sensory mismatch, albeit to a lesser degree because of the vestibular stimulation provided by the movement. Cirio et al. [11] proposed a hybrid movement method which supported physical walking within the physical boundaries of a room but, once near the edges, the user could extend his/her hand to initiate rate-based virtual traversal in the direction of pointing, again potentially suffering from sensory mismatch.

\section{Redirected Walking}

Redirected walking utilizes imperceptible lateral shifts in the visual scene that cause the user to walk in an arc while believing they are walking straight ahead (e.g., $[18,36,43,44])$. This facilitates longer virtual walking routes than straight trajectories, allowing users to walk through an effectively infinite virtual space. Research has sought to measure how sensitive users are to the subtle rotations (and occasionally movement compressions [44]) that induce redirected walking. Steinicke et al. [42-44] found that users could be redirected by up to $17-18^{\circ}$ over a $5 \mathrm{~m}$ walking distance without being reliably aware, representing an arc with a $32 \mathrm{~m}$ diameter. If users are engaged in a secondary task (i.e., not focused on detecting movement discrepancies), Hodgson et al. [19] found that they can be redirected along an arc of only $15.3 \mathrm{~m}$ diameter without becoming aware, and curvature and translational gain can be implemented concurrently without users noticing [15]. In these large spaces, perceptual illusions $[8,47]$, change blindness [9] or visual distractors [10,39] can be used to reduce a user's awareness of manipulations. However, regular manipulation of the virtual scene may interfere with tasks or cognitive processing, and redirection algorithms may require knowledge of intended destinations. If it is not necessary for the user to be unaware of any discrepancies, arcs of only $6.6 \mathrm{~m}$ diameter are feasible [44], but not for free exploration.

The seminal redirected walking research (e.g., [42-44]) mapped physically curved paths to virtual straight paths to test the illusion, but more recent work has looked at the tolerance for detection of curved physical paths, either simply walking through a room [28], or in conjunction with touching a wall surface $[32,33]$. The research suggests that there are larger tolerances (i.e., lowered sensitivity) to variations in the curvature of physical vs. virtual paths, compared to straight physical vs. curved virtual paths, and curved walls can be perceived as straight walls [32,33]. Langbehn et al. [29] reduced the necessary physical space to $5 \times 5 \mathrm{~m}$ by utilising curved paths, but this relied on users following preset paths. 
Efforts have also gone into facilitating multiple users in redirected walking environments [2]. Azmandian et al. [1] recently published a general-purpose "Redirected Walking Toolkit" to support development and deployment of redirected walking setups. For a review of redirected walking and other techniques see Vasylevska et al. [53].

There are significant limitations to using redirected walking, principally its incompatibility with free exploration of the virtual space coupled with the large physical spaces required. Redirected walking steers the user away from physical walls, steering which requires relatively straight walking paths and often knowledge of an intended destination. It cannot fully support a user who may need to make sudden or unpredictable movements in any direction throughout the space, without resorting to re-orientation events which break immersion. Also, it currently requires a space that can house a walking arc with a diameter of at least $\sim 7 \mathrm{~m}$ [44], or $15 \mathrm{~m}$ for less constrained exploration [19]. These dimensions are too large for environments where consumer VR systems are used (the average UK living room is only $4.8 \mathrm{~m}^{2}$ [41]). It is also not yet known how redirected walking may impact interaction in VR.

\section{Impossible Spaces}

Rather than utilising continuous imperceptible shifts to the position of the user in the VE, "impossible spaces" [47], or simply 'overlapping spaces', utilise change blindness to alter the physical layout of a space outside of the user's field of view (FOV). For example, a user sees a virtual room and, after walking into it, the layout of the space behind them is changed so that the door is in a new position, directing them into more open space [46,51] or, upon leaving a room, the user is redirected back to the same physical space where a different virtual room is now shown [52]. Alternatively, an entirely different room can be loaded behind the user, as an extension of the room currently inhabited [47]. This can be most readily experienced in the video game "Unseen Diplomacy" [49].

This approach has a benefit over redirected walking in that it better supports free exploration of room-scale spaces, however, it is less successful at creating large virtual environments that can be freely explored, and it still relies on manipulating the position and layout of the virtual world, so it must make assumptions about the user's intention before altering the VE. For example, a user may walk into a room past a bookshelf; they then may want to turn around and pick up a book but the software would need to determine if the user wanted a newly-located exit, or to approach the shelf.

\section{Translational Gain}

An alternative method that combines free exploration with smaller spaces, applies a simple gain factor to the physical distance walked, amplifying the amount of virtual translational movement relative to physical movement. Applying a translational gain of, for example, $2 \mathrm{x}, 10 \mathrm{x}$ or $100 \mathrm{x}$ to $1 \mathrm{~m}$ of physical walking results in the user seeing $2 \mathrm{~m}, 10 \mathrm{~m}$ or $100 \mathrm{~m}$ of movement in the VE [21]. The gain is generally applied only to horizontal ( $\mathrm{x}$ - and $\mathrm{z}$-axes) movement, so that vertical 'head-bob' is not amplified, as this can cause nausea [21].

Research has shown that users are unlikely to detect gains of up to $1.6 x[43,44]$, meaning a physical space can be increased by $60 \%$ without the user even being aware of a manipulation. Gains of $2 x$ are noticeable but not problematic $[22,43,44]$, and even large gains of up to 50x can be applied without inducing sickness or negatively affecting spatial orientation $[43,44,57]$, with active engagement in a task reducing the salience of movement mismatch [19]. There is a suggestion that people are more sensitive to accelerated forward/backward movement than along other axes [22]. Zhang et al. [59] showed no difference in the salience of acceleration between a uniform gain and a dynamically ramped up gain. In contrast, Interrante et al. [21] suggested that a uniform gain can cause greater sickness compared to a system called 'seven league boots' which amplifies motion along a single dimension, based on gaze direction and recent movement. However, this is not suitable for free exploration of a space, as movement can be in any horizontal direction, not just forward/backward, and gaze may change rapidly and conflict with walking direction when engaged in virtual tasks.

Crucially, no research has looked at the influence of translational gain on a user's ability to interact with or manipulate objects in the VE. Virtual worlds are not just for looking at or walking through: reaching for, picking up and placing objects are key aspects of interaction. Azmandian et al. [3] used perspective shifts via rotational gain to allow a single haptic object to be used as multiple building blocks. Users were led to reach for (and place) the same physical block while feeling they were picking/placing spatially distributed blocks. However, they were seated, and the technique required the system to know in advance what object the user was going to pick up and where it would be placed. Janeh et al. [23] recently observed that walking biomechanics (gait) are altered under varying conditions of visual flow (translational gain), so it may be that reaching movements are also affected. Our research addresses the limitations of prior work into translational gain by measuring how accurately users can walk up to, reach for, and place objects, under varying levels of translational gain.

\section{OBJECT MANIPULATION UNDER INCREASING TRANSLATIONAL GAIN}

A study was conducted to examine the impact of varying levels of translational gain on a user's ability to reach for and grasp virtual objects. Physical interaction is important for realism [7] and fundamental to creating virtual facsimiles of real-world tasks that feature the same interactions in VR as in reality. Thus, a concrete understanding of the impact of translational gain on object manipulation was required for translational gain to be successfully applied to general purpose interactive VR. To examine this in a controlled manner, we designed a task that included phases of both walking around virtual obstacles and reaching for virtual objects at varying heights and positions. In doing so, we incorporated 
a range of common reaching movements, such as bending/crouching down, reaching to various heights, and stretching over a table.

\section{Physical Space}

We used an empty $3.5 \mathrm{~m} * 3.5 \mathrm{~m}$ office space and applied simple gain levels to all horizontal ( $\mathrm{x}$ - and $\mathrm{z}$ - axis) translational movement: $1.0 \mathrm{x}$ (1-to-1 movement), $1.5 \mathrm{x}, 1.75 \mathrm{x}, 2.0 \mathrm{x}$ and $3.0 \mathrm{x}$, resulting in square virtual spaces with a length and breadth of $3.5 \mathrm{~m}, 5.25 \mathrm{~m}, 6.125 \mathrm{~m}, 7 \mathrm{~m}$ and $10.5 \mathrm{~m}$ from the same physical space. We used an HTC Vive [20] for tracking the head and hands and providing the visual display. The experiment took place in a $6 \mathrm{~m} \times 5 \mathrm{~m}$ office in the School but we obtained optimal tracking accuracy with the Vive using a $3.5 \mathrm{~m} * 3.5 \mathrm{~m}$ space in the centre of the room. The cable for the Vive ran down to the floor and across to the PC running the software. An experimenter continually monitored participants so that they did not trip on the cable or lose their balance. Participants also held and manipulated the cable using his/her non-dominant hand.

\section{Virtual Environment}

The VE (Figure 1) consisted of a warehouse with floor, walls, ceiling lights and structural pillars. The experiment made use of between $3.5 \mathrm{~m} * 3.5 \mathrm{~m}$ (no translational gain) and $10.5 \mathrm{~m} * 10.5 \mathrm{~m}(3.0 \mathrm{x}$ gain) in the centre of the $20 \mathrm{~m} \times 30 \mathrm{~m}$ virtual room, depending on the condition. Target arrays were placed at each of the four edges of this central space, in either a horizontal table configuration or a vertical wall configuration (see Figure 2). Participants were instructed to walk to a highlighted wall/table, pick up a highlighted target, then walk to the opposite wall/table and place the target in the highlighted position. They were instructed to stand directly in front of the walls/tables when reaching.

\section{Target Arrays}

Each target was a red cube, measuring $5 \mathrm{~cm}^{3}$ with an image of a green arrow on the side facing the participant. This arrow was used to indicate the orientation that the cube must be in when placing it. Cubes were separated from each other by $1 \mathrm{~cm}$ on all sides, values that were arrived at based on pilot testing. To perform a grab/release, users would align a visual attach point on the controller to the cube being targeted. This attach point was a transparent version of the same cube (see Figure 3 ). The target size and separation balanced a need for precise targeting (not too big) and robust target selection. While the arrays contained many cubes, only 9 were used as targets to be picked up and placed down, to keep the total
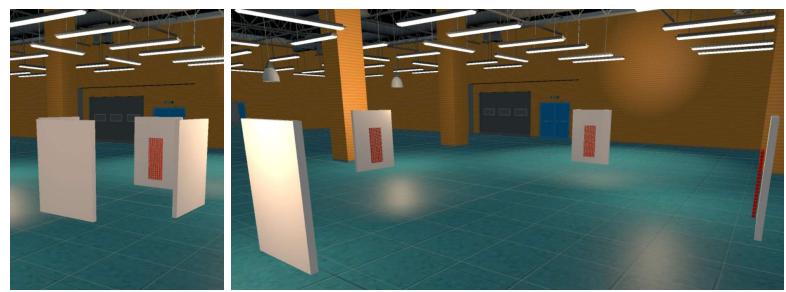

Figure 1: Left: Virtual environment with 1.0x (no) gain. Right: Virtual environment with 3.0x gain.
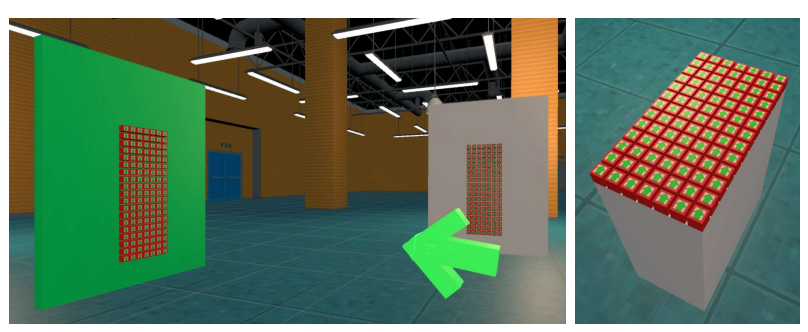

Figure 2: Left: Highlighted wall for next target. Right: Table.

task time to a reasonable length. Entirely randomising the target location within the array could lead to unequal reaching locations/actions across participants so we fixed the positions of the 9 targets (see Walls and Tables below).

We employed two target array configurations, walls and tables, for several reasons: 1) they closely approximate object placements on shelves and tables; 2) walls allow us to see the effects of reaching vertically, and tables the effects of reaching horizontally; 3) as only horizontal translation is amplified (for reasons of comfort), picking from a vertical array is potentially less susceptible to movement gain, as the participant can squat vertically, remaining still horizontally, then reach for a target, all the while experiencing no amplification. In the table configuration, reaching inherently involves horizontal movement. The number of visible boxes in the array varied between the walls and tables for practical reasons explained below, but both used the same number of targets.

Walls

Shelving (and cupboards) at home and in shopping environments are positioned from the floor up to above shoulder height for easy access. To avoid unbalancing participants by forcing them reach too high, the top row of the wall array was positioned at shoulder height, measured and set per-participant at the start of each experiment. The bottom row of the array was approximately at shin height, to ensure a wide reaching range. There were 19 rows $x 7$ columns in the wall array, and the positions of the 9 target cubes were in columns (from top left) 2, 4 and 6 on rows 2, 10 and 18. Using rows 10 and 18 allowed us to measure the effect on accuracy and subjective reports of small and large downward-reaching movements. The total width of the array was limited such that those targets on the outer columns could be reached without leaning or stepping. Only the dominant hand was used for reaching, as requiring participants to reach across their bodies would be uncomfortable and awkward.
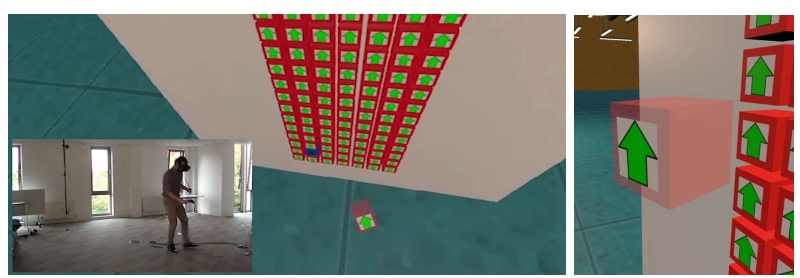

Figure 3: Left: User reaching to select target cube, with inset real-world environment. Right: Alignment cube. 


\section{Tables}

As the tables were used to test horizontal reaching, the height was set at hip-height, again measured per-participant. A higher table would make distant reaching more awkward, and a lower table would introduce vertical reaching. It was not possible to reach as far as 19 rows on a table, and so we limited the number to 13 (with row 1 nearest the participant): a value arrived at from pilot testing. This required a large but feasible (and not unbalancing) reaching movement. Each row again consisted of 7 columns, and the 9 target cubes were again placed in columns 2,4 and 6 , only this time on rows 2,7 and 12 .

\section{Obstacles}

To add complexity to the walking part of the task, and to measure the effect of amplified translation on movement, waist-height virtual obstacles were placed in the space between walls/tables which the participant had to walk around. These obstacles were placed so as to require a straight line, a left-right zig-zag or a parabolic curve (see Figure 4). The positions of the curve and zig-zag obstacles were mirrored to require an equal number of movements to the left and right.

\section{Experimental Task}

The experimental task required the participant to walk to one wall/table, pick up a highlighted cube from the rack, turn around and walk to the opposite wall/table and place the cube in a designated place in the rack, walking a route to avoid the virtual obstacles. Participants completed both a wall-only and a table-only condition at each level of translation gain. The Vive controller was not rendered in the virtual environment (nor was the hand), but the controller's location was indicated by a partially transparent red target cube, acting as a guide where participants should match the position and orientation of the transparent cube with the target cube.

Participants started each condition with their backs to the 'north' virtual wall, facing into the centre of the space. At random, the cube rack on either the left or right wall (at $45^{\circ}$ angle) was visually highlighted (changed from grey to green) and an arrow appeared in the middle of the room, pointing to the highlighted rack (see Figure 2). This indicated that the participant should walk to that rack (with no obstacle). When they were within a radius of $1 \mathrm{~m}$ of the rack in virtual space one of the 9 target cubes was visually highlighted in blue. The participant then reached for and picked up the cube using the Vive controller, by matching the position of the transparent controller cube with the target cube and activating the controller trigger button. The controller cube then changed from transparent to opaque.

The participant then turned $180^{\circ}$ and walked towards the opposite cube rack (now highlighted green), avoiding the obstacles now placed on the floor. When within a $1 \mathrm{~m}$ radius of the opposite wall, one of the 9 target positions within the rack was highlighted in blue, indicating the participant was to place the held cube in that location, and in the same orientation (matching the green arrow), by positioning the cube using the controller and pressing the trigger button. Once done,
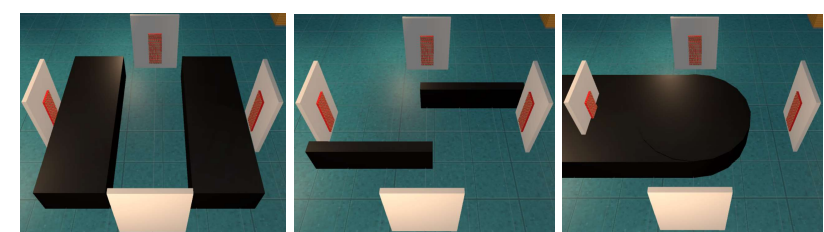

Figure 4: The three walking routes. Left: Straight. Middle: Zig-zag. Right: Curve. Obstacles were scaled based on gain.

the participant again turned $180^{\circ}$ to face into the virtual room and the next trial began (either left or right side highlighted). During each condition, each of the 9 target positions was picked from, and placed into, three times in a random order. The obstacles were each applied to one of the three trials of each target, in a random order.

\section{Movement Amplification Levels}

We tested five levels of translational gain: 1.0x (1-to-1 movement), $1.5 \mathrm{x}, 1.75 \mathrm{x}, 2.0 \mathrm{x}$ and $3.0 \mathrm{x}$, resulting in virtual spaces with a length and breadth of $3.5 \mathrm{~m}, 5.25 \mathrm{~m}, 6.125 \mathrm{~m}$, $7 \mathrm{~m}$ and $10.5 \mathrm{~m}$ respectively. Research has shown that amplifications up to $2 \mathrm{x}$ are only slightly noticeable when walking $[22,43,44]$, and we wanted to identify how similar amplifications affected reaching. While amplifications of $10 \mathrm{x}$ or even $50 \mathrm{x}$ have been studied before, they were done so only in the context of movement, with no interaction. Pilot testing showed such high levels made interaction extremely challenging.

\section{Experimental Design}

The experiment had a five (Translational Gain) x two (Array Orientation) factor within-subjects design: all participants completed one wall and one table condition under all levels of translational gain, resulting in 10 conditions in total. The order of Gain levels was counterbalanced across participants, and the order of Orientations was counterbalanced across Gain levels.

To minimise any cumulative effects of fatigue and nausea, participation was split into two sessions separated by 3 or 4 days: the first session comprised of 6 conditions ( 3 Gain levels $x 2$ Orientations), and the second session the remaining 4 conditions (2 Gains $\times 2$ Orientations). Within each condition, 9 target locations were selected from 3 times, resulting in 27 trials. Twenty healthy participants ( 11 female, 9 male) took part, aged 20 to 35 (mean 24.7), and were paid £20 upon completing the second session. Participants were primarily staff and students from the University, and were screened for any physical or non-corrected visual impairment.

\section{Measures}

We collected objective task performance measures, subjective workload, comfort responses, and open-ended interview responses about the experience as a whole.

\section{Task Measures}

- Time: Trial Duration (including both picking and placing and walking to walls/tables) and Reaching Time (from arriving in $1 \mathrm{~m}$ virtual proximity to target); 
- Movement: Walking Velocity, Tortuosity (a measure of bend in a line, in this case it is the mean of the degree of change of bearing between successive points across the trajectory of the walking route in the $\mathrm{x} / \mathrm{z}$ axes);

- Reaching precision: Target Selection Correctness (whether the correct cube was picked up or placed), Target Selection Accuracy (the Euclidean distance between the centres of the target and selected cube).

\section{Subjective Responses}

- Nausea: Simulator Sickness Questionnaire (SSQ) [24];

- Workload: NASA TLX [16];

- 7-point Likert scales, from "Strongly Disagree" (1) to "Strongly Agree" (7): "My movement felt unusual"; "I felt unstable while walking"; "I felt unstable while reaching".

\section{RESULTS}

For all results, a repeated measures ANOVA was performed. Where data were non-parametric, an Aligned-Rank Transform [56] was used to allow use of parametric methods. For post hoc contrasts, the lsmeans [31] R package was used with Tukey adjustment. Where Violin plots [17] are used, they display a rotated kernel density plot on either side of a box plot, allowing for density estimation, with notched box plots denoting the $95 \%$ CI [26].

\section{Reaching accuracy and task performance}

Hereafter we will refer to both the target grab and target release phases as the same event: a target selection. If we examine target selection correctness, all conditions had mean correctness of $>90 \%$, with no significant differences between 1. $\mathrm{x}$ up to $2.0 \mathrm{x}$ (see Table 1). However, there was a significant difference between 3.0x Gain and the other levels, with mean accuracy dropping to $90 \%(\mathrm{sd}=12 \%)$ for Table and $85 \%$ $(\mathrm{sd}=12 \%)$ for Wall.

Selection accuracy also significantly decreased for 3.0x Gain compared to all other conditions, with the notable increase in dispersal of selection points present in Figure 5. Target orientation accuracy was not significantly affected by Gain, but it was significantly affected by array orientation: Wall targets were misaligned by an average of $30^{\circ}$.

Mean trial duration significantly increased with Gain for all combinations except $1.75 \mathrm{x}-2.0 \mathrm{x}$, taking $\sim 12$ seconds at $1.0 \mathrm{x}$ versus $\sim 16$ seconds at $3.0 \mathrm{x}$. Whilst this would appear a notable increase across what are like-for-like tasks, this increase is largely accounted for when we consider a side-effect of

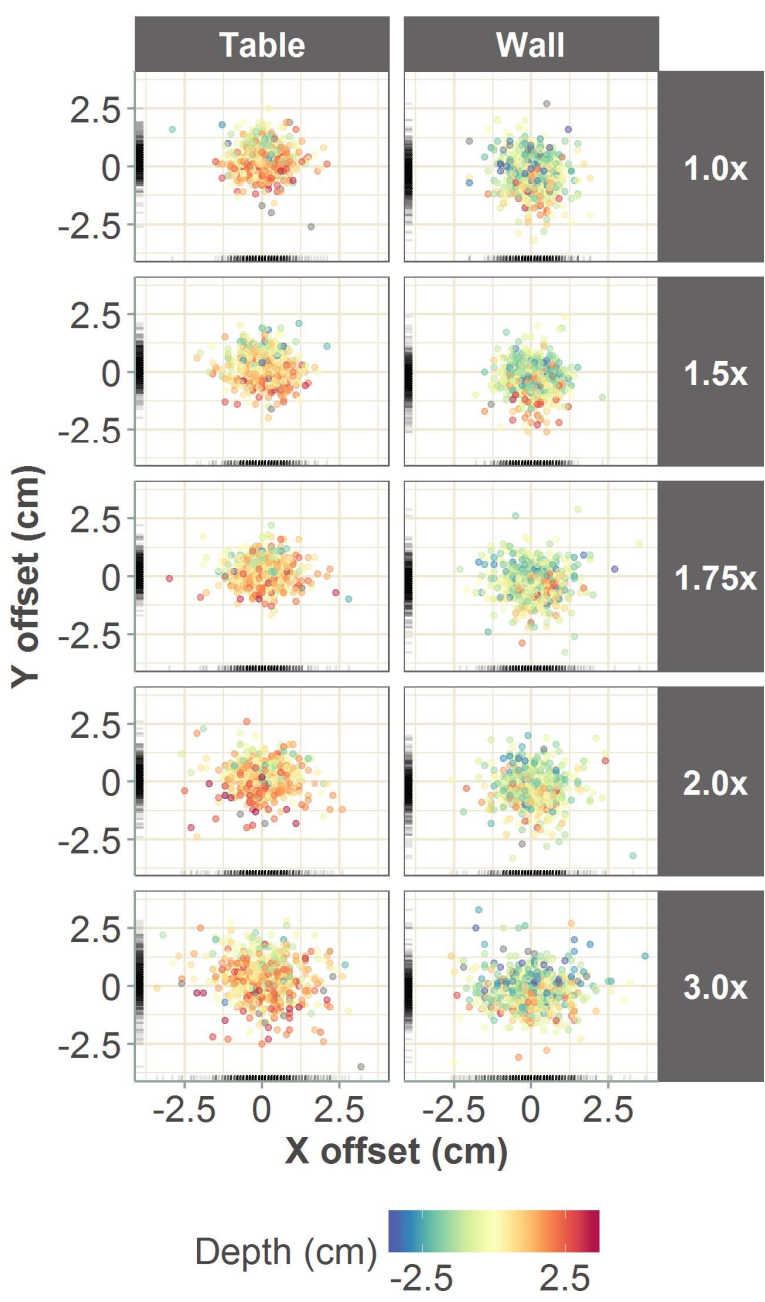

Figure 5. Scatterplot of controller attach point at target grab/release, front-on for Wall, top-down for Table (meaning both are effectively front-on relative to the target). The spread increases with increasing gain.

\begin{tabular}{|c|c|c|c|c|}
\hline Measure & Gain factor & Gain post hoc $(\mathrm{p}<0.05)$ & Orientation factor & Gain x Orientation \\
\hline $\begin{array}{l}\text { Target correct- } \\
\text { ness }\end{array}$ & $F(4,171)=5.74, p<0.01$ & $\{1.5 \mathrm{x}, 1.75 \mathrm{x}, 2.0 \mathrm{x}\}-3.0 \mathrm{x}$ & $\mathrm{F}(1,171)=0.68, \mathrm{p}=0.41$ & $\mathrm{~F}(4,171)=2.23, \mathrm{p}=0.07$ \\
\hline $\begin{array}{l}\text { Selection accu- } \\
\text { racy }\end{array}$ & $\mathrm{F}(4,171)=7.55, \mathrm{p}<0.01$ & $\{1.0 \mathrm{x}, 1.5 \mathrm{x}, 1.75 \mathrm{x}, 2.0 \mathrm{x}\}-3.0 \mathrm{x}$ & $\mathrm{F}(1,171)=1.84, \mathrm{p}=0.18$ & $\mathrm{~F}(4,171)=0.057, \mathrm{p}=0.99$ \\
\hline $\begin{array}{l}\text { Orientation ac- } \\
\text { curacy }\end{array}$ & $F(4,171)=0.90, p=0.47$ & NA & $\mathrm{F}(1,171)=180.16, \mathrm{p}<0.05$ & $\mathrm{~F}(4,171)=1.15, \mathrm{p}=0.34$ \\
\hline Trial duration & $F(4,171)=62.91, p<0.01$ & All except $1.75 x-2.0 x$ & $F(1,171)=0.56, p=0.46$ & $\mathrm{~F}(4,171)=0.95, \mathrm{p}=0.44$ \\
\hline $\begin{array}{l}\text { Reaching dura- } \\
\text { tion }\end{array}$ & $\mathrm{F}(4,171)=6.99, \mathrm{p}<0.01$ & $\begin{array}{l}1.0 x-1.5 x \\
\{1.5 x, 1.75 x, 2.0 x\}-3.0 x\end{array}$ & $\mathrm{~F}(1,171)=0.02, \mathrm{p}=0.89$ & $\mathrm{~F}(4,171)=2.17, \mathrm{p}=0.08$ \\
\hline
\end{tabular}

Table 1. Quantitative performance measures: Target correctness, a binary measure of whether centre of the controller attach point was within the volume of the target; Selection accuracy, the Euclidean distance between the controller attach point and the centre of the target; Orientation accuracy, the minimum angle between the orientation of the controller attach point and the centre of the target; Trial duration, the time taken to walk to the first target, grab it, walk around obstacle, and then place it; Reaching duration, the time taken to grab/place once within 1 metre of the table/wall. Significant differences are highlighted. 
reaching for targets under translational gain: in the increased gain conditions the effective position of the virtual target in the real world varies based on the arm/reach length of the user reaching for the target. If we consider a point in reality $1.75 \mathrm{~m}$ away, this point would also be $1.75 \mathrm{~m}$ away in VR at $1.0 x$ gain. Given an arm reach of $0.5 \mathrm{~m}$, then the user would have to walk $1.25 \mathrm{~m}$, to within a $0.5 \mathrm{~m}$ proximity of the target, to be able to reach out and touch it.

Now consider this scenario at $3.0 \mathrm{x}$ gain. The same point at $1.75 \mathrm{~m}$ away in reality would be $5.25 \mathrm{~m}$ away in VR. With an arm reach of $0.5 \mathrm{~m}$, the user would have to walk $4.75 \mathrm{~m}$ in VR space to be able to reach out to the target, which would mean walking $4.75 / 3.0 \mathrm{x}=1.58 \mathrm{~m}$, compared to $1.25 \mathrm{~m}$ under $1.0 \mathrm{x}$ gain. In effect, the user would have to walk further to reach out and touch the target, even though if they were standing at the $1.75 \mathrm{~m}$ mark in reality, they would be standing on the target under both $1.0 \mathrm{x}$ and $3.0 \mathrm{x}$ gain. This is because their hand position is not accelerated in the way that their head position is. Assuming a reach of $0.5 \mathrm{~m}$, this difference amounts to a $26 \%$ increase on the effective distance in reaching for the target at 3.0x gain versus 1.0x gain, explaining most of the difference between the $\sim 12 \mathrm{~s}$ trial time at $1.0 \mathrm{x}$ and the $\sim 16 \mathrm{~s}$ at $3.0 \mathrm{x}$.

Consequently, utilizing measures of distance and time when reaching for targets under translational gain is problematic. However, there was a significant increase in the time taken to select the target once within $1 \mathrm{~m}$ virtually of the Table/Wall between the 3.0x gain and the other levels (see Reaching duration, Table 1), even though the user would have to move less distance to touch the target. This is indicative of the potential difficulties of reaching for targets in a smaller realworld volume, with the real-world position of the target shifting based on any additional head movement. This can be seen in Figure 6, where the fixed position of the target evident in

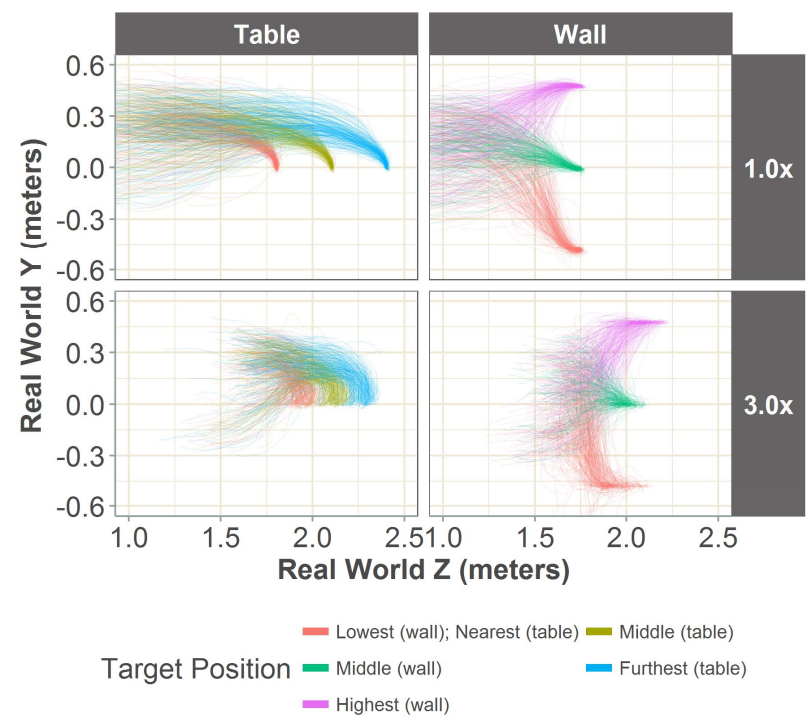

Figure 6. Side-on view of reach trajectory in real-world space when within $1 \mathrm{~m}$ virtually of the target. the $1.0 \mathrm{x}$ condition degrades significantly in the $3.0 \mathrm{x}$ condition, with the ballistic phase of the reach compacted into smaller physical bounds.

\section{Walking movement}

Given the variance in distance to the target based on both gain and user reach length, velocity was instead used to examine the effect of gain on real-world movement. Mean virtual velocity increased significantly for all levels of gain $(\mathrm{F}(4,266)=563.09, \mathrm{p}<0.01$, all contrasts $\mathrm{p}<0.05)$ and across all obstacle types $(\mathrm{F}(2,266)=39.10, \mathrm{p}<0.01$, all contrasts $\mathrm{p}<0.05$ ), with no interactions, going from $\sim 0.6 \mathrm{~m} / \mathrm{s}$ at $1.0 \mathrm{x}$ to $\sim 1.3 \mathrm{~m} / \mathrm{s}$ at $3.0 \mathrm{x}$. This was to be expected, with users moving through the virtual world at an increased rate.

However, the magnitude of this increase in virtual velocity was not proportional with the gain level. Indeed, there was also a significant effect on real-world velocity by gain $(F(4,266)=101.79, p<0.01$, all contrasts $p<0.01$ except $1.75 x$ $-2.0 \mathrm{x})$ and obstacle type $(\mathrm{F}(2,266)=40.80, \mathrm{p}<0.01$, all contrasts $\mathrm{p}<0.01)$ with no interactions. As can be seen in Figure 7 , real-world velocity decreased by $\sim 25 \%$ from approximately $\sim 0.6 \mathrm{~m} / \mathrm{s}$ at $1.0 \mathrm{x}$ to $\sim 0.45 \mathrm{~m} / \mathrm{s}$ at $3.0 \mathrm{x}$. In effect, users self-moderated their walking pace as gain increased and as they navigated the winding paths of the curve and zig-zag obstacles. This reinforces findings that suggest users perform an unconscious gain compensation [37].

The steadiness of walking movement was also examined by calculating the tortuosity of the participants' movements on the $x / z$ axes (i.e. from above). Ignoring obstacle type (as each obstacle has a different required path tortuosity), a significant effect was found for gain $(F(4,266)=20.66, p<0.01)$, with contrasts indicating differences between $1.0 \mathrm{x}-1.75 \mathrm{x}$, $\{1.0 x, 1.5 x\}-2.0 x$, and $\{1.0 x, 1.5 x, 1.75 x, 2.0 x\}-3.0 x$. However, given the decrease in real-world walking velocity as gain increased, we cannot accurately state whether this is indicative of unsteady movement, or confounded to some unknown degree by a change in step count resulting from the

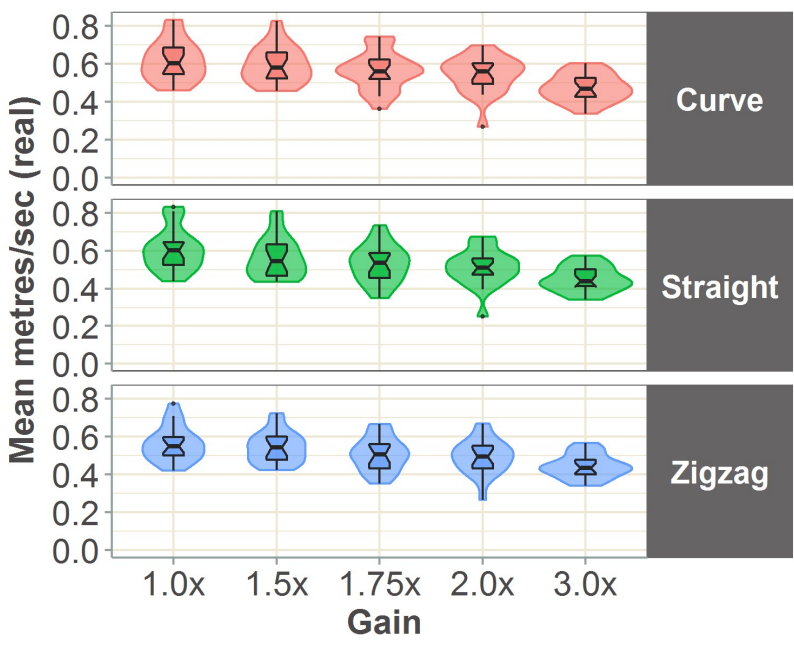

Figure 7. Real-world walking velocity whilst navigating obstacles mid-trial. 
change in velocity. For example, if step count increased, this would lead to additional gait phase changes (i.e. increased side-to-side head movement), which would be impair this measure.

\section{Subjective Responses}

As can be seen in Table 2, there were significant main effects of gain on both overall TLX workload (see Figure 8) and on all the TLX subscales, with differences predominantly arising between $\{1.0 \mathrm{x}, 1.5 \mathrm{x}, 1.75 \mathrm{x}, 2.0 \mathrm{x}\}$ and $3.0 \mathrm{x}$. 3.0x gain appears to be the point at which performance decreases and workload increases. With respect to the SSQ simulator sickness score, again 3.0x marks a boundary upon which there is a significant difference relative to all other conditions. However, the score is still comparatively low, with 3.0x gain having a mean of $\sim 21$ compared to a mean of $\sim 10$ for $1.0 \mathrm{x}$. This is just past the threshold (18.8) that Kennedy et al. [24] suggested indicated a problematic level of sickness for a simulator, with the means for all other conditions below this threshold. Regarding the three Likert-type questions, again there were repeated significant differences between 3.0x gain and all other levels. For unstable walking and unstable reaching there were also differences between the lower levels of gain and 2.0x gain.

\section{Interview Responses}

Short, loosely guided interviews were conducted at the end of the study asking about any particular difficulties or unpleasant aspects of the study, with interviews coded using Initial Coding (participants' statements were assigned emergent codes over repeated cycles). These codes were grouped using a thematic approach and reported based on frequency and interest, with representative excerpts quoted.

Bending down to reach was problematic

It was noted by 11 participants that bending down to reach for targets under translational gain, particularly in reference

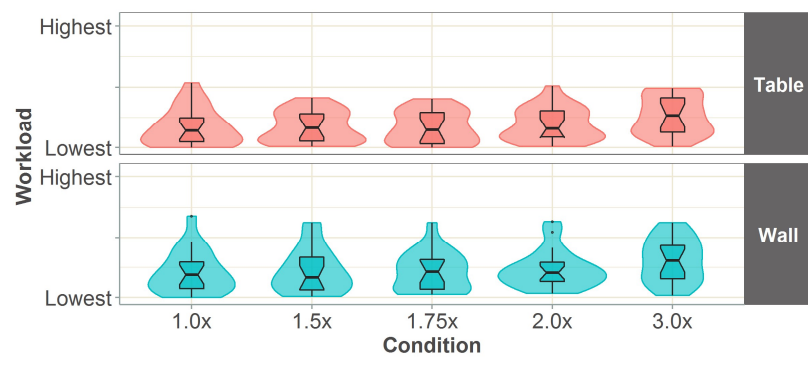

Figure 8. Overall NASA TLX workload score across different levels of gain.

to the lowest Wall target, was problematic in terms of stability:

P1: "Reaching down when it's the walls, I felt really uncomfortable, really "blurgh", really unstable. Especially when it was too fast."

P16: "In the (conditions) that felt more accelerated it was sometimes uncomfortable to reach and pick things up that were lower down. But not in the less accelerated ones."

P19: "When I was reaching to grab something on the lower shelf I felt like the whole thing was moving.

Three participants explicitly noted that they adapted how they reached for the target by crouching rather than bending, thus reducing the translational gain experienced by minimizing head movement throughout the reach:

P3: "Under higher acceleration, moving my head back and forward felt very unusual, so after I realized that, I started crouching down [rather than bending over].

P14: "It felt like it didn't quite match my physical motion and um... when reaching down - absolutely uncomfortable. Because I literally had to sometime go into a squat in order to stabilize myself."

\begin{tabular}{|c|c|c|c|c|}
\hline Measure & Gain factor & Gain post hoc $(\mathrm{p}<0.05)$ & Type factor & Gain x Type \\
\hline TLX workload & $F(4,171)=14.62, p<0.01$ & $\{1.0 x, 1.5 x, 1.75 x, 2.0 x\}-3.0 x$ & $F(1,171)=17.12, p<0.01$ & $\mathrm{~F}(4,171)=0.05, \mathrm{p}=1$ \\
\hline TLX effort & $F(4,171)=7.93, p<0.01$ & $\{1.0 x, 1.5 x, 1.75 x, 2.0 x\}-3.0 x$ & $\mathrm{~F}(1,171)=8.60, \mathrm{p}<0.01$ & $\mathrm{~F}(4,171)=0.09, \mathrm{p}=0.99$ \\
\hline TLX frustration & $\mathrm{F}(4,171)=8.51, \mathrm{p}<0.01$ & $\begin{array}{l}1.0 \mathrm{x}-2.0 \mathrm{x} \\
\{1.0 \mathrm{x}, 1.5 \mathrm{x}, 1.75 \mathrm{x}\}-3.0 \mathrm{x}\end{array}$ & $F(1,171)=3.99, p=0.047$ & $\mathrm{~F}(4,171)=0.12, \mathrm{p}=0.97$ \\
\hline TLX mental demand & $F(4,171)=9.79, p<0.01$ & $\{1.0 x, 1.5 x, 1.75 x, 2.0 x\}-3.0 x$ & $F(1,171)=8.38, p<0.01$ & $\mathrm{~F}(4,171)=0.65, \mathrm{p}=0.63$ \\
\hline TLX physical demand & $F(4,171)=3.93, p<0.01$ & $1.0 \mathrm{x}-3.0 \mathrm{x}$ & $\mathrm{F}(1,171)=26.01, \mathrm{p}<0.01$ & $\mathrm{~F}(4,171)=0.20, \mathrm{p}=0.93$ \\
\hline TLX performance & $F(4,171)=7.99, p<0.01$ & $\{1.0 x, 1.5 x, 1.75 x\}-3.0 x$ & $\mathrm{~F}(1,171)=7.69, \mathrm{p}<0.01$ & $\mathrm{~F}(4,171)=0.74, \mathrm{p}=0.56$ \\
\hline TLX temporal demand & $\mathrm{F}(4,171)=3.67, \mathrm{p}<0.01$ & $1.75 \mathrm{x}-3.0 \mathrm{x}$ & $\mathrm{F}(1,171)=1.01, \mathrm{p}=0.32$ & $\mathrm{~F}(4,171)=0.09, \mathrm{p}=0.98$ \\
\hline $\begin{array}{l}\text { SSQ simulator sickness } \\
\text { score }\end{array}$ & $\mathrm{F}(4,171)=8.55, \mathrm{p}<0.01$ & $\{1.0 \mathrm{x}, 1.5 \mathrm{x}, 1.75 \mathrm{x}, 2.0 \mathrm{x}\}-3.0 \mathrm{x}$ & $F(1,171)=1.43, p=0.23$ & $\mathrm{~F}(4,171)=0.45, \mathrm{p}=0.77$ \\
\hline Unusual movement & $\mathrm{F}(4,171)=15.51, \mathrm{p}<0.01$ & $\begin{array}{l}\{1.0 x, 1.5 x\}-2.0 x \\
\{1.0 x, 1.5 x, 1.75 x, 2.0 x\}-3.0 x\end{array}$ & $\mathrm{~F}(1,171)=0.60, \mathrm{p}=0.44$ & $\mathrm{~F}(4,171)=0.23, \mathrm{p}=0.92$ \\
\hline Unstable whilst walking & $\mathrm{F}(4,171)=25.31, \mathrm{p}<0.01$ & $\begin{array}{l}\{1.0 \mathrm{x}, 1.5 \mathrm{x}, 1.75 \mathrm{x}\}-2.0 \mathrm{x} \\
\{1.0 \mathrm{x}, 1.5 \mathrm{x}, 1.75 \mathrm{x}, 2.0 \mathrm{x}\}-3.0 \mathrm{x}\end{array}$ & $F(1,171)=0.18, p=0.68$ & $\mathrm{~F}(4,171)=0.63, \mathrm{p}=0.64$ \\
\hline Unstable whilst reaching & $\mathrm{F}(4,171)=20.34, \mathrm{p}<0.01$ & $\begin{array}{l}1.0 \mathrm{x}-1.75 \mathrm{x} \\
\{1.0 \mathrm{x}, 1.5 \mathrm{x}\}-2.0 \mathrm{x} \\
\{1.0 \mathrm{x}, 1.5 \mathrm{x}, 1.75 \mathrm{x}, 2.0 \mathrm{x}\}-3.0 \mathrm{x}\end{array}$ & $\mathrm{F}(1,171)=1.71, \mathrm{p}=0.19$ & $\mathrm{~F}(4,171)=1.03, \mathrm{p}=0.39$ \\
\hline
\end{tabular}

Table 2. Questionnaire responses for NASA TLX, Simulator Sickness Questionnaire, and unusual movement ("My movement in the virtual environment felt unusual", higher is worse), stability whilst walking ("I felt unstable while walking") and stability whilst reaching ("I felt unstable while reaching"). Significant differences are highlighted. 
P20: "When you had the plane (wall) if you had to bend that was the worst. I always had to find a stable stance from which to operate. I never really bent down as I would normally to pick something up. "'

Inconsistent perception of space and gain

The translational gain conditions appeared to warp perception of the size of the physical space for 4 participants, to the extent that the $1.0 \mathrm{x}$ gain condition felt smaller/slower than reality, an observed phenomenon with 1:1 walking in VR $[4,14]$ :

P2: "The smaller environments felt a wee bit strange, it felt like I was taking $3 / 4$ of the steps I was actually taking. "

P16: "I felt like the smaller ones were smaller than the actual room and the bigger ones were bigger than the actual room - none felt like the actual size of the room."

High gain affected stability

For 4 participants, high gain affected the steadiness in VR whilst moving:

P13: "I just noticed the difference in the amount you moved with each step. I guess that affected how stable the VR felt. If it was further away, if you moved your head it was more jittery - there was more extreme motion. When things were closer it was a lot tighter and felt a lot nicer."

\section{DISCUSSION}

This study set out to examine both the effect of translational gain on reaching accuracy and the impact of object manipulation on the perception and usability of translational gain. Firstly, 3.0x gain was repeatedly shown to be statistically less accurate, and exhibit significantly greater workload, than the other tested levels. Considering target accuracy, up to $2.0 \mathrm{x}$ gain is usable. It may be possible to go higher but we do not know at what point accuracy degrades between $2.0 \mathrm{x}$ and 3.0x gain. Whilst reaching behaviour is subtly different between $1.0 \mathrm{x}$ and the increased gain conditions, this did not impair task performance measurably. When we consider the effect that repeated object manipulations had on the user experience of translational gain, we can begin to rule out any levels of gain beyond 2.0x more categorically. Simulator sickness scores indicated a hard cap at 2.0x, beyond which an increased level of sickness was experienced. The TLX scores also show that beyond $2 \mathrm{x}$ gain the workload increased significantly.

The perception of movement instability questions were inline with research suggesting that $1.6 x$ gain was the threshold of imperceptibility, with movement at $2.0 \mathrm{x}$ being noted as unusual compared to $\{1.0 \mathrm{x}, 1.5 \mathrm{x}\}$. Instability in movement was noted for $\{1.0 \mathrm{x}, 1.5 \mathrm{x}, 1.75 \mathrm{x}\}$ versus $2.0 \mathrm{x}$, with significant instability in reaching between $1.0 \mathrm{x}$ and $1.75 \mathrm{x}$.

Accordingly, we begin to see there are many factors which can inform what is the maximal level of translational gain that can be accommodated. Given we are using a subset of gain levels, we can state the range within which a measure degrades. Based on the ranges in Table 3, we would suggest

\begin{tabular}{lcc}
\hline Measure & $\begin{array}{c}\text { Current usable } \\
\text { maximum gain }\end{array}$ & $\begin{array}{c}\text { Gain becomes } \\
\text { problematic at }\end{array}$ \\
\hline Accuracy & $2.0 \mathrm{x}$ & $3.0 \mathrm{x}$ \\
Simulator sickness & $2.0 \mathrm{x}$ & $3.0 \mathrm{x}$ \\
Frustration & $1.75 \mathrm{x}$ & $2.0 \mathrm{x}$ \\
"Unusual" movement & $1.75 \mathrm{x}$ & $2.0 \mathrm{x}$ \\
Instability (movement) & $1.75 \mathrm{x}$ & $2.0 \mathrm{x}$ \\
Instability (reaching) & $1.5 \mathrm{x}$ & $1.75 \mathrm{x}$ \\
\hline
\end{tabular}

Table 3. Guidelines for applying translational gain. The true "problematic" max will lie somewhere between the values in the "usable" and "problematic" columns.

that translational gain of $1.5 \mathrm{x}$ would represent a safe value if the VR simulation is to involve dynamic reaching and bending to interact with graspable objects. It is worth noting that the task required continuous and repeated walking, reaching and rotation actions from participants. Tasks which require less frequent movements and adjustments may well allow larger acceptable gains. For VR experiences with infrequent object interactions and limited-to-no bending/reaching, $1.75 x$ gain would be an acceptable level. Training and practice may also reduce the negative effects of larger gain levels, as these values could be marginally increased, as the true maximums remain somewhere between the safe and problematic points.

\section{Future Research}

Our results give a strong starting point for the choice of gain level in a VE. However, different scenarios may need different levels. Examining different virtual tasks with different levels of interactivity would more concretely establish the acceptable level of gain based on the type and frequency of the virtual interactions the user is expected to perform. In the case of translational gain, it would appear that as the vestibular system is sensing the "correct" motion in terms of direction and orientation, differences between the visual and vestibular systems in terms of the magnitude of motion are less problematic. As has been noted by VR use in-car [34] however, visual perception of motion in VR currently brings with it a number of caveats. For example, the field of view of current consumer headsets is typically $\sim 110^{\circ}$ - in effect, our visual system is receiving only a subset of the information it could potentially process, with peripheral motion cues almost entirely absent. Decreasing the field of view has been noted to decrease simulator sickness in the case of vection [13]. Thus, conversely, it would be reasonable to hypothesise that as the field of view of consumer VR HMDs increases and provides our visual system with more information, the perception of translational gain may vary.

Our perception of motion is dependent on a variety of visual cues linked to perception of depth [40]. It would thus be reasonable to hypothesize that different virtual environments may elicit different effects. For example, consider a jungle scene packed with foliage. The perception of the movement 
of close foliage might result in different recommended levels of gain. Given the variety of scenes that can be portrayed, this presents a problematic factor to take into account, but research has found that visual density does not strongly affect sensitivity to rotational gain in redirected walking [38]. Further research using different VEs would establish if this holds for translational gain too.

Attention should be paid to bending/reaching under translational gain. As our participant interviews emphasized, the acts of bending over or stretching to reach for an object were problematic because the participants' feet are rooted to the ground, but they still experience translational movement, which we term the "sliding floors" effect. This is because the translation is applied to the position of the user's head (as it is the VR HMD which is tracked). This also results in a magnification of postural sway: as the user's head moves left and right, the visual magnitude of this movement will be increased. Whilst filtering can be used to correct this [35], if translation was applied to the user's centre of gravity, or the centre-point between the user's feet, then this problem would be diminished. This suggests alternative forms of tracking would be needed. We have performed some initial experimentation to this end, testing a "body-tracked" translational gain implementation by tracking the position of an HTC Vive controller attached to the small of the user's back. However, there are significant tracking and ergonomic issues to be overcome if such a solution is to be viable. With better tracking, the upper bound of acceptable translational gain could be increased.

Finally, there is the aspect of habituation to translational gain. It could be expected that, given gradual small increases in gain over time, users might overcome inaccuracy in reaching and become hardened to symptoms of simulator sickness. These increases could, for example, be based on duration of exposure and duration since last exposure per user, meaning that more frequent users might find the virtual boundaries of their games and applications slowly increasing over time. In such a scenario, the recommendations made in this paper may simply represent starting points for exposure, rather than end points of maximal gain. However, a means of assessing the suitability of a given gain increase would need to be devised and integrated into consumer VR experiences, whilst the impact of longitudinal exposure to translational gain is as-yet unknown.

\section{Summary}

Our results show that translational gain can now be applied to highly interactive VR experiences. This paper was motivated by our underlying aim of aiding people with Acquired Brain Injury $(\mathrm{ABI})$ to practice real-world tasks safely through VR. To do so, we needed a general-purpose approach that would create the largest fully explorable/reachable interactive virtual room possible from a small physical space, accommodating the physical limitations of the homes and facilities these experiences might be deployed within. We wanted to do this whilst retaining physical walking as the means of locomotion, as opposed to other methods that require training and explicit user interactions (such as pointing a controller to teleport), to ensure there was still a physical cost to actions in VR, and to preserve spatial understanding. Only by satisfying these constraints could we have the basis for creating virtual facsimiles of real-world tasks and environments that do not introduce additional confounds based on the method of exploring the virtual space. We would suggest that, given our guidelines, translational gain satisfies these constraints.

We provide guidelines for the application of translational gain to VR, as well as a code snippet that will allow for translational gain to be applied to any SteamVR scene whilst preserving the play area bounds and scaling/moving objects appropriately (see https://gist.github.com/markmcg/ff2215824cc503b40ee9800810753068). Translational gain is not necessarily the best tool for every $\mathrm{VE}$, but it is a tool whose use should be considered alongside the state-ofthe-art in redirected walking, locomotion and omni-directional treadmills. It can provide immediate benefits to practitioners in creating low-cost interactive virtual rooms that are fully explorable and comparable in dimensions to livingrooms, kitchens and shops, based on much smaller physical room-scale spaces.

\section{CONCLUSIONS}

The physical space available for room-scale Virtual Reality (VR) is limited by common home or office spaces. Utilising translational gain, the size of fully explorable virtual environments can be quickly and easily expanded with existing hardware. For the first time, this research has shown how these movement amplifications impact common object interactions such as reaching and picking. This paper has explored how the picking and placing of virtual objects in VR is affected by translational gains of up to $3.0 \mathrm{x}$ the physical movement. We found that reaching accuracy is maintained at up to $2 \mathrm{x}$ gain, however, going beyond this diminishes accuracy and increases simulator sickness and perceived workload. We have provided guidelines regarding how translational gain can be utilized by practitioners without compromising the VR user's capability to interact with their virtual surroundings.

\section{ACKNOWLEDGMENTS}

This work was funded by the EPSRC IAA University of Glasgow VET Project.

\section{REFERENCES}

1. Mahdi Azmandian, Timofey Grechkin, Mark Bolas, and Evan Suma. 2016. The redirected walking toolkit: a unified development platform for exploring large virtual environments. 2016 IEEE 2nd Workshop on Everyday Virtual Reality (WEVR): 9-14. https://doi.org/10.1109/WEVR.2016.7859537

2. Mahdi Azmandian, Timofey Grechkin, and Evan Suma Rosenberg. 2017. An evaluation of strategies for twouser redirected walking in shared physical spaces. Proceedings - IEEE Virtual Reality: 91-98. 
https://doi.org/10.1109/VR.2017.7892235

3. Mahdi Azmandian, Mark Hancock, Hrvoje Benko, Eyal Ofek, and Andrew D. Wilson. 2016. Haptic Retargeting: Dynamic Repurposing of Passive Haptics for Enhanced Virtual Reality Experiences. Proceedings of the 2016 CHI Conference on Human Factors in Computing Systems - CHI '16: 1968-1979.

https://doi.org/10.1145/2858036.2858226

4. T Banton, J Stefanucci, F Durgin, a Fass, and D Proffitt. 2005. The Perception of Walking Speed in a Virtual Environment. Presence 14, 4: 394-406. https://doi.org/10.1162/105474605774785262

5. Doug A. Bowman and Larry F. Hodges. 1997. An evaluation of techniques for grabbing and manipulating remote objects in immersive virtual environments. Proceedings of the 1997 symposium on Interactive $3 D$ graphics - SI3D '97: 35-. https://doi.org/10.1145/253284.253301

6. Evren Bozgeyikli, Andrew Raij, Srinivas Katkoori, and Rajiv Dubey. 2016. Point and Teleport Locomotion Technique for Virtual Reality. In Proceedings of the 2016 Annual Symposium on Computer-Human Interaction in Play - CHI PLAY'16, 205-216. https://doi.org/10.1145/2967934.2968105

7. F.P. Brooks. 1999. What's Real About Virtual Reality? Proceedings IEEE Virtual Reality (Cat. No. 99CB36316), December: 2-3. https://doi.org/10.1109/VR.1999.756916

8. Gerd Bruder, Frank Steinicke, Benjamin Bolte, Phil Wieland, Harald Frenz, and Markus Lappe. 2013. Exploiting perceptual limitations and illusions to support walking through virtual environments in confined physical spaces. Displays 34, 2: 132-141. https://doi.org/10.1016/j.displa.2012.10.007

9. Gerd Bruder, Frank Steinicke, and Phil Wieland. 2011. Self-motion illusions in immersive virtual reality environments. 2011 IEEE Virtual Reality Conference: 39-46. https://doi.org/10.1109/VR.2011.5759434

10. Haiwei Chen and Henry Fuchs. 2017. Supporting free walking in a large virtual environment. Proceedings of the Computer Graphics International Conference on CGI '17: 1-6. https://doi.org/10.1145/3095140.3095162

11. Gabriel Cirio, Maud Marchal, Tony Regia-Corte, and Anatole Lécuyer. 2009. The magic barrier tape: a novel metaphor for infinite navigation in virtual worlds with a restricted walking workspace. Proceedings of the 16th ACM Symposium on Virtual Reality Software and Technology - VRST'09 1, 212: 155. https://doi.org/10.1145/1643928.1643965

12. Rudolph P. Darken, William R. Cockayne, and David Carmein. 1997. The omni-directional treadmill: A Locomotion Device for Virtual Worlds. Proceedings of the 10th annual ACM symposium on User interface software and technology - UIST '97: 213-221. https://doi.org/10.1145/263407.263550

13. Ajoy S. Fernandes and Steven K. Feiner. 2016. Combating VR sickness through subtle dynamic fieldof-view modification. In 2016 IEEE Symposium on 3D User Interfaces, 3DUI 2016 - Proceedings, 201-210. https://doi.org/10.1109/3DUI.2016.7460053

14. Harald Frenz, Markus Lappe, Marina Kolesnik, and Thomas Bührmann. 2007. Estimation of travel distance from visual motion in virtual environments. $A C M$ Transactions on Applied Perception 4, 1: 3-es. https://doi.org/10.1145/1227134.1227137

15. Timofey Grechkin, Jerald Thomas, Mahdi Azmandian, Mark Bolas, and Evan Suma. 2016. Revisiting detection thresholds for redirected walking. Proceedings of the ACM Symposium on Applied Perception - SAP '16: 113-120. https://doi.org/10.1145/2931002.2931018

16. SG Hart and LE Staveland. 1988. Development of NASA-TLX (Task Load Index): Results of empirical and theoretical research. In Human mental workload. Retrieved June 17, 2013 from http://humanfactors.arc.nasa.gov/groups/TLX/download s/NASA-TLXChapter.pdf

17. JL Hintze and RD Nelson. 1998. Violin plots: a box plot-density trace synergism. The American Statistician. Retrieved November 26, 2015 from $\mathrm{http} / / /$ amstat.tandfonline.com/doi/abs/10.1080/0003130 5.1998.10480559

18. Eric Hodgson and Eric Bachmann. 2013. Comparing four approaches to generalized redirected walking: simulation and live user data. IEEE transactions on visualization and computer graphics 19, 4: 634-43. https://doi.org/10.1109/TVCG.2013.28

19. Eric Hodgson, Eric Bachmann, and David Waller. 2011. Redirected walking to explore virtual environments. ACM Transactions on Applied Perception 8, 4: 1-22. https://doi.org/10.1145/2043603.2043604

20. HTC. HTC Vive. Retrieved September 18, 2017 from http://www.htcvr.com/

21. Victoria Interrante, Brian Ries, and Lee Anderson. 2007. Seven league boots: A new metaphor for augmented locomotion through moderately large scale immersive virtual environments. IEEE Symposium on 3D User Interfaces 2007 - Proceedings, 3DUI 2007: 167-170. https://doi.org/10.1109/3DUI.2007.340791

22. P. M. Jaekl, M. R. Jenkin, and Laurence R. Harris. 2005. Perceiving a stable world during active rotational and translational head movements. Experimental Brain Research 163, 3: 389-399. https://doi.org/10.1007/s00221-004-2191-8

23. Omar Janeh, Eike Langbehn, Frank Steinicke, Gerd Bruder, Alessandro Gulberti, Monika Poetter, O Janeh, E Langbehn, and F Steinicke. 2017. Walking in Virtual 
Reality: Effects of Manipulated Visual Self-Motion on Walking Biomechanics. ACM Trans. Appl. Percept. ACM Transactions on Applied Perception 14, 12: 1-15. https://doi.org/10.1145/3022731

24. Robert S. Kennedy, Norman E. Lane, Kevin S. Berbaum, and Michael G. Lilienthal. 1993. Simulator Sickness Questionnaire: An Enhanced Method for Quantifying Simulator Sickness. The International Journal of Aviation Psychology 3, 3: 203-220. https://doi.org/10.1207/s15327108ijap0303_3

25. Behrang Keshavarz, Bernhard E Riecke, Lawrence J Hettinger, and Jennifer L Campos. 2015. Vection and visually induced motion sickness: how are they related? Frontiers in psychology 6: 472. https://doi.org/10.3389/fpsyg.2015.00472

26. Martin Krzywinski and Naomi Altman. 2014. Points of Significance: Visualizing samples with box plots. Nature Methods 11, 2: 119-120. https://doi.org/10.1038/nmeth.2813

27. Andreas Kunz, Markus Zank, Morten Fjeld, and Thomas Nescher. 2016. Real walking in virtual environments for factory planning and evaluation. Procedia CIRP 44: 257-262. https://doi.org/10.1016/j.procir.2016.02.086

28. Eike Langbehn, Paul Lubos, Gerd Bruder, and Frank Steinicke. 2017. Bending the Curve: Sensitivity to Bending of Curved Paths and Application in RoomScale VR. IEEE Transactions on Visualization and Computer Graphics 23, 4: 1349-1358. https://doi.org/10.1109/TVCG.2017.2657220

29. Eike Langbehn, Paul Lubos, Gerd Bruder, and Frank Steinicke. 2017. Application of redirected walking in room-scale VR. Proceedings - IEEE Virtual Reality 2: 449-450. https://doi.org/10.1109/VR.2017.7892373

30. "Leap Motion.” 2018. VR Platform. leapmotion.com. Retrieved January 4, 2018 from https://www.leapmotion.com/product/vr

31. RV Lenth. Least-squares means: the R package lsmeans. jstatsoft.org. Retrieved September 13, 2017 from https://www.jstatsoft.org/article/view/v069i01/v69i01.p df

32. Keigo Matsumoto, Yuki Ban, Takuji Narumi, Yohei Yanase, Tomohiro Tanikawa, and Michitaka Hirose. 2016. Unlimited corridor: redirected walking techniques using visuo haptic interaction. ACM SIGGRAPH 2016 Emerging Technologies on - SIGGRAPH '16: 1-2. https://doi.org/10.1145/2929464.2929482

33. Keigo Matsumoto, Takuji Narumi, Yuki Ban, Tomohiro Tanikawa, and Michitaka Hirose. 2017. Turn physically curved paths into virtual curved paths. Proceedings IEEE Virtual Reality: 247-248. https://doi.org/10.1109/VR.2017.7892269

34. Mark Mcgill, Alexander Ng, and Stephen Brewster.
2017. I Am The Passenger: How Visual Motion Cues Can Influence Sickness For In-Car VR. Proceedings of the 2017 CHI Conference on Human Factors in Computing Systems - CHI '17: 5655-5668. https://doi.org/10.1145/3025453.3026046

35. Thomas Nescher and Andreas Kunz. 2013. Using Head Tracking Data for Robust Short Term Path Prediction of Human Locomotion. . Springer, Berlin, Heidelberg, 172-191. https://doi.org/10.1007/978-3-642-388033_10

36. Nguyen Thi Anh Ngoc, Yannick Rothacher, Peter Brugger, Bigna Lenggenhager, and Andreas Kunz. 2016. Estimation of individual redirected walking thresholds using standard perception tests. In Proceedings of the 22nd ACM Conference on Virtual Reality Software and Technology - VRST '16, 329-330. https://doi.org/10.1145/2993369.2996304

37. Anh Nguyen, Federico Cervellati, and Andreas Kunz. 2017. Gain Compensation in Redirected Walking. 4. https://doi.org/10.1145/3139131.3139167

38. Anders Paludan, Jacob Elbaek, Mathias Mortensen, Morten Zobbe, Niels Christian Nilsson, Rolf Nordahl, Lars Reng, and Stefania Serafin. 2016. Disguising rotational gain for redirected walking in virtual reality: Effect of visual density. Proceedings - IEEE Virtual Reality 2016-July: 259-260. https://doi.org/10.1109/VR.2016.7504752

39. Tabitha C. Peck, Henry Fuchs, and Mary C. Whitton. 2012. The design and evaluation of a large-scale realwalking locomotion interface. IEEE Transactions on Visualization and Computer Graphics 18, 7: 10531067. https://doi.org/10.1109/TVCG.2011.289

40. David Regan, K I Beverley, and Max Cynader. 1979. The visual perception of motion in depth. Scientific American 241, 1: 136-51. https://doi.org/10.2307/24965248

41. Bibiana Da Silva. 2017. The average dimensions of a living room. ehow.co.uk. Retrieved September 18, 2017 from http://www.ehow.co.uk/info_8574543_averagedimensions-living-room.html

42. F Steinicke, G Bruder, J Jerald, H Frenz, and M Lappe. 2010. Estimation of Detection Thresholds for Redirected Walking Thechniques. IEEE Transactions on Visualization and Computer Graphics 16, 1: 17-27. https://doi.org/10.1109/TVCG.2009.62

43. Frank Steinicke, Gerd Bruder, Jason Jerald, Harald Frenz, Markus Lappe, and Harald Frenz. 2008. Analyses of Human Sensitivity to Redirected Walking. Vrst 146, 3: 149-156. https://doi.org/10.1145/1450579.1450611

44. Frank Steinicke, Gerd Bruder, Timo Ropinski, and Klaus Hinrichs. 2008. Moving Towards Generally Applicable Redirected Walking. Proceedings of the 10th Virtual Reality International Conference (VRIC 2008): 
$15-24$.

45. Evan A. Suma, Mahdi Azmandian, Timofey Grechkin, Thai Phan, and Mark Bolas. 2015. Making small spaces feel large: infinite walking in virtual reality. In $A C M$ SIGGRAPH 2015 Emerging Technologies on SIGGRAPH '15, 1-1. https://doi.org/10.1145/2782782.2792496

46. Evan A. Suma, Seth Clark, David Krum, Samantha Finkelstein, Mark Bolas, and Zachary Warte. 2011. Leveraging Change Blindness for Rediection in Virtual Environments. In Proceedings - IEEE Virtual Reality, 159-166. https://doi.org/10.1109/VR.2011.5759455

47. Evan A. Suma, Zachary Lipps, Samantha Finkelstein, David M. Krum, and Mark Bolas. 2012. Impossible spaces: Maximizing natural walking in virtual environments with self-overlapping architecture. IEEE Transactions on Visualization and Computer Graphics 18, 4: 555-564. https://doi.org/10.1109/TVCG.2012.47

48. Sam Tregillus, Majed Al Zayer, and Eelke Folmer. 2017. Handsfree Omnidirectional VR Navigation using Head Tilt. Proceedings of the 2017 CHI Conference on Human Factors in Computing Systems - CHI '17: 4063-4068. https://doi.org/10.1145/3025453.3025521

49. Triangular Pixels. 2017. Unseen Diplomacy: a VR obstacle course. triangularpixels.net. Retrieved December 30, 2017 from http://www.triangularpixels.net/cms/games/unseendiplomacy/

50. Martin Usoh, Kevin Arthur, Mary C Whitton, Rui Bastos, Anthony Steed, Mel Slater, and Frederick P Brooks. 1999. Walking $>$ walking-in-place $>$ flying, in virtual environments. Proceedings of the 26th annual conference on Computer graphics and interactive techniques - SIGGRAPH '99: 359-364. https://doi.org/10.1145/311535.311589

51. Khrystyna Vasylevska and Hannes Kaufmann. 2017. Towards efficient spatial compression in selfoverlapping virtual environments. 2017 IEEE Symposium on 3D User Interfaces, 3DUI 2017 Proceedings: 12-21. https://doi.org/10.1109/3DUI.2017.7893312

52. Khrystyna Vasylevska, Hannes Kaufmann, Mark Bolas, and Evan A. Suma. 2013. Flexible spaces: Dynamic layout generation for infinite walking in virtual environments. IEEE Symposium on $3 D$ User Interface 2013, 3DUI 2013 - Proceedings: 39-42. https://doi.org/10.1109/3DUI.2013.6550194

53. Khrystyna Vasylevska, Iana Podkosova, and Hannes Kaufmann. 2015. Walking in Virtual Reality: Flexible Spaces and Other Techniques. In The Visual Language of Technique, L. Cocchiarella (ed.). Springer, 81-97. https://doi.org/10.1007/978-3-319-05341-7

54. Wiki. 2017. Locomotion - Virtual Reality and
Augmented Reality Wiki - VR and AR Wiki. XinReality. Retrieved September 13, 2017 from https://xinreality.com/wiki/Locomotion

55. Preston Tunnell Wilson, William Kalescky, and Ansel Maclaughlin. 2015. VR Locomotion : Walking > Walking in Place $>$ Arm Swinging. 243-249.

56. Jacob O. Wobbrock, Leah Findlater, Darren Gergle, and James J. Higgins. 2011. The aligned rank transform for nonparametric factorial analyses using only anova procedures. In Proceedings of the 2011 annual conference on Human factors in computing systems CHI '11, 143. https://doi.org/10.1145/1978942.1978963

57. Xianshi Xie, Qiufeng Lin, Haojie Wu, Gayathri Narasimham, Timothy P. McNamara, Thomas H. Carr, John Rieser, and Bobby Bodenheimer. 2010. A System for Exploring Large Virtual Environments That Combines Scaled Translational Gain and Interventions. Symposium on Applied Perception in Graphics and Visualization 1, 212: 65-72. https://doi.org/10.1145/1836248.1836260

58. Catherine Zanbaka, Benjamin Lok, Sabarish Babu, Dan Xiao, Amy Ulinski, and Larry F. Hodges. 2004. Effects of travel technique on cognition in virtual environments. Proceedings - Virtual Reality Annual International Symposium: 149-156. https://doi.org/10.1109/VR.2004.1310068

59. Ruimin Zhang, Bochao Li, and Scott A Kuhl. 2014. Human Sensitivity to Dynamic Translational Gains in Head-Mounted Displays. In Proceedings of SUI 2014, 62-65. https://doi.org/10.1145/2659766.2659783 